\title{
Hydranencephaly: A rare case report
}

\author{
Nalini Sharma ${ }^{1}$, Vinayak Jante ${ }^{2}$, Rituparna Das ${ }^{1}$, Subrat Panda ${ }^{3}$, Mandeep Sagar ${ }^{4}$ \\ From ${ }^{1}$ Assistant Professor, ${ }^{2}$ Senior Resident, ${ }^{3}$ Associate Professor, Department of OBG, ${ }^{4}$ Senior Resident, Department of Radiology, North Eastern \\ Indira Gandhi Regional Institute of Health and Medical Sciences, Shillong, Meghalaya, India
}

\begin{abstract}
Hydranencephaly (HE) is a rare condition occurring in $<1 / 10,000$ births worldwide. It is one of the recognized forms of brain malformations that are usually associated with intrauterine fetal demise rarely seen in postnatal life. HE can often be misdiagnosed due to certain common features with other neurological abnormalities such as hydrocephalus, holoprosencephaly, and porencephaly. Here, we report the case of a 26-year-old pregnant patient at 34 weeks who was referred with ultrasonography finding of HE which was confirmed by fetal MRI. The decision to deliver the baby was taken expecting an extremely poor outcome after discussing with the family.
\end{abstract}

Key words: Cerebrospinal fluid, Hydranencephaly, Hydrocephalus, Internal carotid artery occlusion

$\mathrm{H}$ ydranencephaly (HE) is a rare condition occurring in $<1 / 10,000$ births worldwide. It is an isolated abnormality with a severe prognosis [1]. It is one of the most severe forms of bilateral cerebral cortical anomaly, affecting more than one-third of all children. In this condition, the cerebral hemispheres are replaced by a membranous sac filled with cerebrospinal fluid, glial tissue, and ependyma. However, however, there will be the preservation of the skull. There is variable involvement of the inferior frontal, temporal, and occipital lobes. The midbrain, cerebellum, thalami, and basal ganglia are usually not involved. HE can affect only one hemisphere, which is called hemihydranencephaly, which is even rarer than $\mathrm{HE}$ and may have a better prognosis [2]. $\mathrm{HE}$ is one of the recognized forms of brain malformations usually associated with intrauterine fetal demise rarely seen in postnatal life. It can often be misdiagnosed due to certain common features with other neurological abnormalities such as hydrocephalus, holoprosencephaly, and porencephaly [2]. The exact etiology is not known. The most common cause is found to be occlusion of the supra-clinoid part of the internal carotid artery (ICA), which leads to infarction of cerebral structures [3]. So far, no sex or racial preference has been discovered [1].

The rationale of our case report is to report on this condition, utilizing data from the literature, and also present our experience with a pregnant patient who presented in her third trimester with bilateral fetal HE.

\section{Access this article online}

Received - 11 October 2021

Initial Review - 29 October 2021

Accepted - 09 November 2021

DOI: $10.32677 /$ ijcr.v7i11.3136

\section{CASE REPORT}

A 26-year-old Primigravida at 34 weeks of gestation was referred to the Department of Obstetrics and Gynecology of our institute. In view of pre-eclampsia and bilateral fetal $\mathrm{HE}$, her marriage was nonconsanguineous. There was no family history of genetic or congenital anomalies. She did not have a regular antenatal check-up and did not perform routine appointment scans, anomaly scans, or growth scans on the fetus during pregnancy. She had her first antenatal visit in the third trimester at 33 weeks at a private hospital where she was diagnosed with pre-eclampsia and on antenatal ultrasound, the fetus was found to have bilateral HE. She was referred in view of the same condition to our institute for a second opinion.

A physical examination at admission revealed blood pressure of $150 / 102 \mathrm{mmHg}$, heart rate of 92 beats/min, and respiratory rate of $20 \mathrm{breaths} / \mathrm{min}$. Ophthalmic and other systemic (central nervous system, cardiovascular system) examinations were within normal limits.

Initial laboratory tests showed normal hemoglobin $(13 \mathrm{~g} / \mathrm{dL})$ and platelet count $(200 \times 109 / \mathrm{L})$. The evaluation of coagulation parameters was within normal limits. Her biochemical analysis for renal and hepatic function showed total protein $(5.60 \mathrm{~g} / \mathrm{dL})$, albumin $(2.8 \mathrm{~g} / \mathrm{dL})$, albumin/globulin ratio (1.14), proteinuria $(++)$, normal transaminases (ALT 23.2 IU/L, AST 29.2 IU/L), and lactate dehydrogenase (LDH 233 IU/L). Blood pressure was well controlled with the tablet Labetalol $100 \mathrm{mg}$ twice a day.

Obstetric ultrasound was repeated and the findings corresponded with the previous ultrasound that is bilateral HE.

Correspondence to: Dr. Vinayak Jante, Room No: S-15, RMO Hostel, North Eastern Indira Gandhi Regional Institute of Health and Medical Sciences, Shillong, Meghalaya, India. E-mail: vjante1819@gmail.com

(C) 2021 Creative Commons Attribution-NonCommercial 4.0 International License (CC BY-NC-ND 4.0). 
Although ultrasound is good enough for the diagnosis of HE, on patients' request to confirm the diagnosis and also to differentiate between other close differentials such as hydrocephaly and holoprosencephaly, fetal Magnetic resonance imaging (MRI) was done, which revealed fetal HE and deformed posterior structures (Fig. 1).

After consulting with the family, the decision was made to deliver the baby at 34 weeks and 5 days, with the expectation of an extremely poor outcome. She was planned for induction of labor with a pharmacological method. The duration of induction was prolonged, requiring 6 doses of 50 microgram Misoprostol given at a 6-h interval. She delivered a female fresh stillborn baby of $1.9 \mathrm{~kg}$ and had a normal physical appearance. The head of the baby was of normal size (head circumference $32 \mathrm{~cm}$ ). The postpartum period was uneventful and blood pressure was controlled with tablets of Labetalol $100 \mathrm{mg}$ twice a day, and the patient was discharged on day 3 .

\section{DISCUSSION}

HE is characterized by the absence of cerebral hemispheres which are replaced by cerebrospinal fluid and necrotic debris, covered by leptomeninges. It is an encephaloclastic abnormality. The midbrain, thalamus, basal ganglia may be preserved, and the flax cerebri is usually present within the skull [3].

The exact etiopathogenesis of HE is still not known, so several theories have been postulated. However, the most accepted theory as suggested by many researchers is brain damage caused by early involvement of ICAs which is suggested by i) angiographic and autoptic observations where both aplastic and hypoplastic arteries were reported in ICAs and ii) the anatomic distribution follows the ICA supply [2]. Mayers did an experiment on monkeys to describe the etiology of HE. In his study, monkey fetuses at different gestational ages were subjected to ligation of the bilateral ICAs and jugular veins. The fetuses were then restored to the uterus and delivered at term. An examination of baby monkey brains revealed HE due to vascular shutdown predominantly when carried out during earlier gestational age.

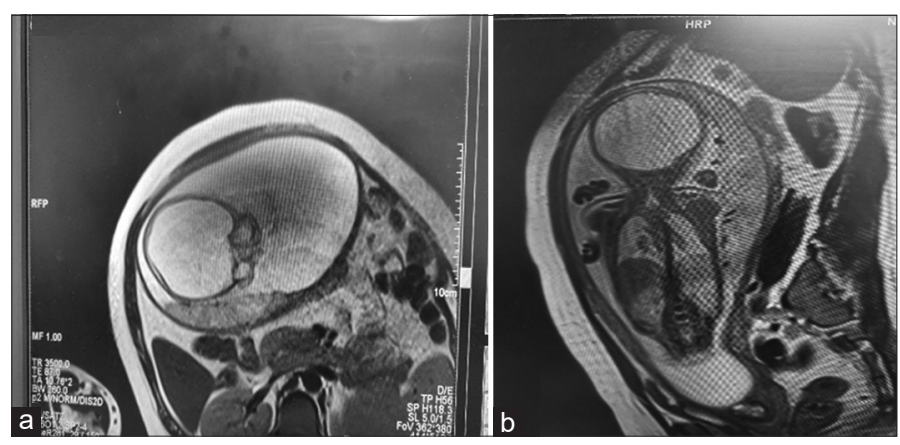

Figure 1: (a) Axial T2 weighted non-contrast MRI of the fetus shows replacement of the supra and infratentorial structures by fluid signal intensity; (b) Sagittal T2 weighted non-contrast MRI of the fetus shows replacement of the supra and infratentorial structures by fluid signal intensity (T2 hyperintense). The falx, bilateral thalami are not visualized, also the posterior fossa structures, spinal cord are deformed and thin cord like. The liquor volume in all the four quadrants were increased, in keeping with polyhydramnios
Some case reports suggest that the occlusion of the ICAs may not be direct as temporary spasm can also lead to ischemic destruction of brain structures [3].

One of the most specific hallmarks of HE on angiography is bilateral ICA occlusion. This sign helps to differentiate between severe hydrocephalus and HE [4]. However, on the other hand, not all cases of HE present with complete occlusion of bilateral ICAs. La Torre and Occhipinti reported a few cases of HE whose findings on angiographic studies showed both anterior and middle cerebral arteries present but stretched as in cases of extreme hydrocephalus [5].

Although the pathogenesis of $\mathrm{HE}$ is thought to be mainly linked to a vascular accident, there are other various and frequently reported aetiologies that may interfere with normal vascular development. Among them, the most reported are intrauterine infections, mainly Toxoplasmosis and other viral infections such as adenovirus, parvovirus, Enterovirus, herpes simplex, EpsteinBarr, cytomegalic, and respiratory syncytial viruses have been implicated in a number of cases. Smoking, cocaine abuse, and estrogen exposure have also been reported [2]. A study done in Israel found that the Akabane virus and Aino virus can cause teratogenic malformations when they infect immunologically naive pregnant women [6]. Either directly or indirectly, all these reported causes can affect the ICAs or other cerebral parts, leading to profound brain alterations. Hence, HE can be considered as a severe pathological event secondary to ischemia of the brain supplied by the ICAs [2].

HE has also been reported in monozygotic twins due to the vascular interchanges between them. This can result in the transfer of intravascular coagulation materials from the deceased co-twin to the surviving twin, causing thromboembolism [3]. Various other congenital anomalies such as Fowler syndrome, arthrogryposis, renal aplastic dysplasia, poly-valvular heart defect, and trisomy 17 have also been associated with HE [7,8].

The ultrasonographic imaging of HE shows the absence of cerebral hemispheres, replaced by homogeneous echogenic material filling the supratentorial space, and the thalami, brain stem, and cerebellum are usually preserved. There is an important difference between the diagnosis of extreme hydrocephaly, alobar holoprosencephaly, and porencephaly [2]. There will be a rim of cortex around the above-mentioned structures in these conditions, so the prognosis may be better than in HE. It is of paramount importance to differentiate HE from other differentials. Subsequently, around $1 \%$ of infants who are initially diagnosed as having hydrocephalus later turn out to be cases of HE [9]. If the hydrocephaly is severe, MRI or a CT scan may be used to make a diagnosis [10].

HE has a very poor prognosis as the majority of brain functions are affected. The affected fetus usually dies in utero or can die at birth. Most infants succumb within 1 year of life. If at all, a child does survive, then they will inevitably be severely handicapped. Most of the cases of HE are diagnosed in the second half of pregnancy, but there are some case reports of diagnosis in the first trimester [3]. It will be depressing for the family due to social and emotional problems that occur after the delivery of a HE. It 
is advisable to help them to be prepared for the potential outcome through proper counseling of the parents regarding the poor prognosis and potential management options [3]. The termination of the pregnancy is recommended once a definitive diagnosis is made due to a poor prognosis. In cases of macrocrania, the term cephalocentesis can be suggested [10].

\section{CONCLUSION}

The findings we described led to the diagnosis of HE, which was confirmed by fetal MRI. Postnatal CT/MRI will be more appropriate, but in our case, it was a stillbirth, so postnatal investigations could not be done. It is critical to diagnose $\mathrm{HE}$ early so that obstetric complications can be avoided and, most importantly, proper parental counseling can be provided. Our case was an unbooked case as she did not have a regular antenatal check-up, so the diagnosis was made in the third trimester. It is challenging for doctors to manage such cases in the late trimester and also very distressful for the family. It is important for preparing the optimal conditions for delivery and allowing for specialized care by pediatricians by early diagnosis of HE.

\section{REFERENCES}

1. Sultana Z, Kumari A, Pathan HG. A rare case of hydranencephaly: A case report. Int J Anat Res 2020;8:7644-8.
2. Pavone P, Praticò AD, Vitaliti G, Ruggieri M, Rizzo R, Parano E, et al. Hydranencephaly: Cerebral spinal fluid instead of cerebral mantles. Ital J Pediatr 2015;40:79-84.

3. Wijerathne BT, Rathnayake GK, Ranaraja SK. A rare variation of hydranencephaly: Case report. F1000Res 2012;1:22.

4. Quek YW, Su PH, Tsao TF, Chen JY, Ng YY, Hu JM, et al. Hydranencephaly associated with interruption of bilateral internal carotid arteries. Pediatr Neonatol 2008;49:43-7.

5. La Torre E, Occhipinti E. Cerebral angiography in hydranencephaly with report of three cases. Eur Neurol 1969;2:184-91.

6. Levin A, Kutznetova L, Kahana R, Rubinstein-Guini M, Stram Y. Highly effective inhibition of Akabane virus replication by siRNA genes. Virus Res 2006;120:121-7.

7. Bendon R, Siddigi J, de Courten-Myers G, Dignan P. Recurrent developmental anomalies: 1. Syndrome of hydranencephaly with renal aplastic dysplasia; 2. Polyvalvular developmental heart defect. Am J Med Genet Suppl 1987;3:357-65.

8. Alasdair GW. Hunter: Brain. In: Stevenson RE, Hall JC. Human Malformations and Relatedanomalies. $2^{\text {nd }}$ ed. New York: Oxford University Press; 2006. p. 639-45.

9. Barozzino T, Sgro M. Transillumination of the neonatal skull: Seeing the light. CMAJ 2002;167:1271-2.

10. Copel J. Obstetric Imaging: Expert Radiology Series. Amsterdam, Netherlands: Elsevier; 2012. p. 238.

Funding: None; Conflicts of Interest: None Stated.

How to cite this article: Sharma $N$, Jante V, Das R, Panda S, Sagar M. Hydranencephaly: A rare case report. Indian J Case Reports. 2021;7(11):491-493. 